\title{
Oxidative decarboxylation of 4-methylthio-2-oxobutyrate by branched-chain 2-oxo acid dehydrogenase complex
}

\author{
Sheelagh M. A. JONES and Stephen J. YEAMAN
}

\begin{abstract}
Highly purified branched-chain 2-oxo acid dehydrogenase complex (BCOADC) oxidizes 4-methylthio2-oxobutyrate and 2-oxobutyrate, with $K_{\mathrm{m}}$ values of $67 \mu \mathrm{M}$ and $18 \mu \mathrm{M}$ respectively. The $V_{\max }$ for oxidation of these substrates is $27 \%$ and $53 \%$ respectively of that for 3-methyl-2-oxobutyrate. Highly purified pyruvate dehydrogenase complex (PDC) oxidizes 2-oxobutyrate $\left(K_{\mathrm{m}} 100 \mu \mathrm{M} ; V_{\max } .49 \%\right.$ of that for pyruvate) but not 4-methylthio-2-oxobutyrate, whereas 2-oxoglutarate dehydrogenase complex will not utilize either 2-oxo acid as substrate. BCOADC kinase is inhibited by both 4-methylthio-2-oxobutyrate and 2-oxobutyrate, with half-maximal inhibition by $45 \mu \mathrm{M}$ and $50 \mu \mathrm{M}$ respectively. Phosphorylation of BCOADC in isolated adipocytes is inhibited by both 4-methylthio-2-oxobutyrate and 2-oxobutyrate, consistent with their inhibitory action of BCOADC kinase. Phosphorylation of PDC is decreased by 2-oxobutyrate, but not by 4-methylthio-2-oxobutyrate.
\end{abstract}

\section{INTRODUCTION}

In addition to its action on the branched-chain 2-oxo acids, highly purified branched-chain 2-oxo acid dehydrogenase complex (BCOADC) also catalyses the oxidative decarboxylation of pyruvate and 2-oxobutyrate [1,2]. The $K_{\mathrm{m}}$ for pyruvate is approx. $1 \mathrm{mM}$, making it unlikely that pyruvate acts as a substrate in vivo, but the $K_{\mathrm{m}}$ for 2-oxobutyrate has been reported to be very similar to the values for the branched-chain 2-oxo acids $[1,2]$.

2-Oxobutyrate is an intermediate in the catabolism of both threonine and methionine. It is formed directly by transamination of threonine and indirectly during the trans-sulphuration pathway of methionine oxidation. However, methionine can also be catabolized via an alternative pathway, involving transamination to 4methylthio-2-oxobutyrate [3]. The relative importance of these two pathways remains controversial.

Evidence is given here that BCOADC catalyses the oxidation of 4-methylthio-2-oxobutyrate and 2-oxobutyrate. Furthermore, these 2-oxo acids are shown to inhibit BCOADC kinase, both the enzyme intrinsic to the purified complex and the kinase in isolated adipocytes.

\section{MATERIALS AND METHODS}

2-Oxo acids were from Sigma and $\left[\gamma_{-32}{ }^{32}\right] A T P$ was from Amersham International. BCOADC was purified from bovine kidney cortex [4]. Pyruvate dehydrogenase complex (PDC) and 2-oxoglutarate dehydrogenase complex (OGDC) were prepared from bovine heart by a minor modification of the method described in [5]. Preparations of PDC were essentially homogeneous, but preparations of OGDC contained contaminating amounts of PDC. Activity due to this contamination was removed by incubation for $30 \mathrm{~min}$ in the presence of $0.2 \mathrm{mM}-\mathrm{ATP}$ and $1.5 \mathrm{mM}-\mathrm{MgCl}_{2}$.

Table 1. Kinetic constants for purified BCOADC and PDC

Enzyme preparation and activity determinations were as described in the Materials and methods section. $K_{\mathrm{m}}$ was calculated by linear-regression analysis of a Lineweaver-Burk plot and is expressed as mean \pm S.E.M., with the number of observations in parentheses. $V_{\max }$ is expressed as a percentage of that attained with 3-methyl-2-oxobutyrate for BCOADC and with pyruvate for PDC. It is expressed as mean \pm S.E.M., for the number of observations in parentheses. Each observation was made with a different preparation of enzyme, and each substrate concentration was assayed in duplicate. Substrates were used at a minimum of five concentrations over the range $0.01-2 \mathrm{~mm}$.

\begin{tabular}{lllc}
\hline Enzyme & \multicolumn{1}{c}{ Substrate } & $K_{\mathrm{m}}(\mu \mathrm{M})$ & Relative $V_{\max }$ \\
\hline \multirow{2}{*}{ BCOADC } & 3-Methyl-2-oxobutyrate & $19 \pm 2(3)$ & $100(4)$ \\
& 4-Methyl-2-oxopentanoate & $21 \pm 5(4)$ & $70 \pm 11(4)$ \\
& 3-Methyl-2-oxopentanoate & $15 \pm 3(3)$ & $41 \pm 5(4)$ \\
& 2-Oxobutyrate & $18 \pm 3(6)$ & $53 \pm 5(4)$ \\
& 4-Methylthio-2-oxobutyrate & $67 \pm 10(5)$ & $27 \pm 3(4)$ \\
PDC & Pyruvate & $32 \pm 1(4)$ & $100(4)$ \\
& 2-Oxobutyrate & $100 \pm 11(4)$ & $49 \pm 7(4)$ \\
& 4-Methylthio-2-oxobutyrate & - & $0(4)$
\end{tabular}

\footnotetext{
Abbreviations used: BCOADC, branched-chain 2-oxo acid dehydrogenase complex; PDC, pyruvate dehydrogenase complex; OGDC, 2-
} oxoglutarate dehydrogenase complex. 
Table 2. Inhibition of BCOADC kinase activity by 2-oxo acids

BCOADC kinase activity was determined in the presence and absence of the 2-oxo acids as described in the Materials and methods section. Maximum inhibition is expressed as mean \pm S.E.M. for five observations and was determined over a concentration range of 0.01-10 mM. Each observation was made with a different preparation of enzyme, and each kinase assay was done in duplicate. The $\mathrm{IC}_{50}$ represents the inhibitor concentration required for half-maximal inhibition of kinase activity.

\begin{tabular}{lcc}
\hline \multicolumn{1}{c}{ Inhibitor } & Maximum inhibition $(\%)$ & $\mathrm{IC}_{\mathbf{5 0}}(\mu \mathrm{M})$ \\
\hline 3-Methyl-2-oxobutyrate & $42 \pm 5$ & 270 \\
4-Methyl-2-oxopentanoate & $59 \pm 4$ & 20 \\
3-Methyl-2-oxopentanoate & $47 \pm 5$ & 60 \\
2-Oxobutyrate & $40 \pm 3$ & 50 \\
4-Methylthio-2-oxobutyrate & $49 \pm 4$ & 45
\end{tabular}

Table 3. Efiect of 2-oxobutyrate and 4-methylthio-2-oxobutyrate on the phosphorylation of the a-subunit of BCOADC and PDC in isolated adipocytes

Cells were incubated in the absence or presence of the 2-oxo acids ( $2.5 \mathrm{~mm})$ for $30 \mathrm{~min}$. Extent of phosphorylation of the $\alpha$-subunit of BCOADC was determined from the integrated area of the corresponding peak on an autoradiograph of an SDS/polyacrylamide gel. The peak areas corresponding to ATP citrate lyase and hormone-sensitive lipase were used as internal standards in each track, to correct for variation in loading. Results are expressed as means \pm S.E..M. for the numbers of observations in parentheses. Statistical significance was determined by paired Student's $t$ test: ${ }^{*} P<0.05,{ }^{* *} P<0.001$, ns not significantly different from control.

\begin{tabular}{llc}
\hline Enzyme & \multicolumn{1}{c}{ Addition } & $\begin{array}{c}\text { Decrease }(\% \text { of control) } \\
\text { in phosphorylation of } \alpha \text {-subunit }\end{array}$ \\
\hline BCOADC & 2-Oxobutyrate & $23 \pm 7(6)^{*}$ \\
& 4-Methylthio-2-oxobutyrate & $24 \pm 7(5)^{*}$ \\
PDC & 2-Oxobutyrate & $66 \pm 6(6)^{* *}$ \\
& 4-Methylthio-2-oxobutyrate & $8 \pm 6(5)^{\mathrm{ns}}$
\end{tabular}

Methods for adipocyte preparation, incubation, preparation of extracts and analysis of phosphoproteins were as described previously $[6,7]$.

The 2-oxo acid dehydrogenase complexes were assayed as described in [1]. BCOADC kinase was assayed at $30^{\circ} \mathrm{C}$ by measuring incorporation of phosphate from $\left[\gamma^{-32}\right.$ P $]$ ATP into protein [8]. BCOADC (containing endogenous kinase) was incubated in $30 \mathrm{~mm}$-imidazole/HCl (pH 7.1)/0.1 mM-EGTA/1.5 mM$\mathrm{MgCl}_{2}$ in a total volume of $50 \mu \mathrm{l}$. The reaction was initiated by addition of $\left[\gamma^{32}\right.$ P $]$ ATP ( 500 c.p.m./pmol) to $0.2 \mathrm{~mm}$ final concentration. Samples $(20 \mu \mathrm{l})$ were taken after $60 \mathrm{~s}$ and treated as in [8], except that the trichloroacetic acid used contained $5 \mathrm{~mm}$-sodium pyrophosphate. Appropriate blanks were included to correct for non-specific binding of radioactivity, and each preparation of enzyme was checked to ensure that the kinase activity was linear with respect to time.

\section{RESULTS}

Highly purified BCOADC oxidizes 4-methylthio-2oxobutyrate with kinetic constants similar to those for oxidation of the branched-chain 2-oxo acids and for 2-oxobutyrate (Table 1). PDC has no detectable activity against 4-methylthio-2-oxobutyrate but, as reported previously [2], it will oxidatively decarboxylate -2oxobutyrate. The $K_{\mathrm{m}}$ and $V_{\max }$. values for the respective substrates are in broad agreement with values reported previously for the complex from liver $[2,9]$, but lower than those reported for the bovine kidney complex [1].
Highly purified OGDC has no detectable activity against 2-oxobutyrate, 4-methylthio-2-oxobutyrate or the branched-chain 2-oxo acids (results not shown).

BCOADC kinase is known to be inhibited by 2-oxo acid substrates $[10,11]$. Table 2 demonstrates that 4-methylthio-2-oxobutyrate and 2-oxobutyrate also inhibit the kinase intrinsic to purified preparations of BCOADC. The maximum extent of inhibition and the concentrations of these compounds necessary for half-maximal inhibition are comparable with the corresponding values for the branched-chain 2-oxo acids. We have demonstrated previously that 2-oxo acid substrates can inhibit phosphorylation of the $\alpha$-subunit of BCOADC in isolated adipocytes [6]. Table 3 shows that 4-methylthio-2-oxobutyrate and 2-oxobutyrate also inhibit phosphorylation of the $\alpha$-subunit in this system. Furthermore, the degree of inhibition is similar to that caused by 3-methyl-2-oxobutyrate and 3-methyl-2-oxopentanoate [6]. The effect of 4-methylthio-2-oxobutyrate is apparently specific for phosphorylation of BCOADC, whereas 2-oxobutyrate inhibits phosphorylation of both BCOADC and the $\alpha$-subunit of PDC, consistent with an involvement of both complexes in oxidation of this metabolite [2].

\section{DISCUSSION}

The data presented here demonstrate that oxidative decarboxylation of 4-methylthio-2-oxobutyrate is catalysed by BCOADC. Furthermore, the kinetic constants for oxidation of 4-methylthio-2-oxobutyrate and 2- 
oxobutyrate are comparable with those for the branchedchain 2-oxo acids. The inability of PDC and OGDC to utilize this compound indicates that its intracellular oxidative decarboxylation is catalysed exclusively by BCOADC. In contrast, the data presented here indicate that both PDC and BCOADC act on 2-oxobutyrate. This is in agreement with recent work from the laboratory of Harris [2].

4-Methylthio-2-oxobutyrate and 2-oxobutyrate are potent inhibitors of BCOADC kinase (Table 2). The kinetics of inhibition of the kinase are complex, and for several inhibitors the curve of inhibition versus inhibitor concentration is apparently biphasic $([11] ; \mathbf{S}$. M. A. Jones \& S. J. Yeaman, unpublished work). In [11] the inhibitory concentration of 2-oxo acid is given as that giving $40 \%$ inhibition $\left(I_{40}\right)$ of the kinase. However, $40 \%$ inhibition is only achieved by some 2-oxo acids at very high concentrations. Therefore we have expressed the potency of each 2-oxo acid in terms of the concentration giving half-maximal inhibition ( IC $\left._{50}\right)$. However, from our data the calculated $I_{40}$ values are in general agreement with those in [11], and the corresponding values for 4-methylthio-2-oxobutyrate and 2-oxobutyrate are approx. $0.2 \mathrm{~mm}$ and $1.5 \mathrm{~mm}$ respectively.

Both 4-methylthio-2-oxobutyrate and 2-oxobutyrate are intermediates in the catabolism of methionine. 2-Oxobutyrate is also formed directly by transamination of threonine. Hence BCOADC is now implicated in the catabolism of five essential amino acids. Furthermore, the 2-oxo acids derived from these amino acids are capable of exerting regulatory control over the specific kinase which phosphorylates that complex.

This work was supported by a project grant from the Medical Research Council, U.K., S. J. Y. is a Lister Institute Research Fellow. We thank Dr. R. C. Honnor for advice on preparation and manipulation of adipocytes.

\section{REFERENCES}

1. Pettit, F. H., Yeaman, S. J. \& Reed, L. J. (1978) Proc. Natl. Acad. Sci. U.S.A. 75, 4881-4885

2. Paxton, R., Scislowski, P. W. D., Davis, E. J. \& Harris, R. A. (1986) Biochem. J. 234, 295-303

3. Livesey, G. (1984) Trends Biochem. Sci. 9, 27-29

4. Lawson, R., Cook, K. G. \& Yeaman, S. J. (1983) FEBS Lett. 157, 54-58

5. Stanley, C. J. \& Perham, R. N. (1980) Biochem. J. 191, 147-154

6. Jones, S. M. A. \& Yeaman, S. J. (1986) Biochem. J. 236, 209-213

7. Honnor, R. C., Dhillon, G. S. \& Londos, C. (1985) J. Biol. Chem. 260, 15122-15129

8. Stepp, L. R., Pettit, F. H., Yeaman, S. J. \& Reed, L. J. (1983) J. Biol. Chem. 258, 9454-9458

9. Parker, P. J. \& Randle, P. J. (1978) Biochem. J. 171, 751-757

10. Lau, K. S., Fatania, H. R. \& Randle, P. J. (1982) FEBS Lett. 144, 57-62

11. Paxton, R. \& Harris, R. A. (1984) Arch. Biochem. Biophys. 231, 48-57 